\title{
Is there a reliable brain morphological signature for migraine?
}

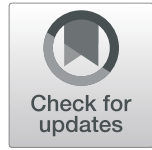

Hong Zhou Wang ${ }^{1}$, Wan Hua Wang ${ }^{1}$, Hai Cun Shi ${ }^{2+}$ and Cong Hu Yuan ${ }^{3 *+}$

\begin{abstract}
Voxel-based morphometry (VBM) is a popular non-invasive magnetic resonance imaging technique to investigate brain gray matter (GM) differences between groups. Recently, two VBM studies in migraine have been published in The Journal of Headache and Pain. Reviewing the two and those previous published VBM studies, we found considerable variations of the results. Spatially diverse brain regions with decreased and increased GM alterations and null findings have been reported. It is interesting to know whether there is a reliable brain morphological signature for migraine. Coordinate-based meta-analysis (CBMA) is increasingly used to quantitatively pool individual neuroimaging studies to identify consistent and reliable findings. Several CBMA have been conducted, however, their results were inconsistent. The algorithms for CBMA have evolved and more eligible VBM studies in migraine have been published. We therefore conducted an updated CBMA using the latest algorithms for CBMA, seed-based $\mathrm{d}$ mapping with permutation of subject images (SDM-PSI). The present CBMA of 32 VBM studies (41 datasets comprising 1252 patients and 1025 healthy controls) found no evidence of consistent GM alterations in migraine. Sensitivity analysis, subgroup meta-analyses, and meta-regression analyses revealed that the result was robust. This negative result indicates that there is no reliable brain morphological signature for migraine. VBM investigations in migraine remain a heterogeneous field. Many potential confounding factors, such as underpowered sample sizes, variations in demographic and clinical characteristics, and differences in MRI scanners, head coils, scanning parameters, preprocessing procedures, and statistical strategies may cause the inconsistences of the results. Future VBM studies are warranted to enroll well-characterized and homogeneous subtype samples with appropriate sample sizes, comprehensively assess comorbidities and medication status, and use well-validated and standardized imaging protocols and processing and analysis pipelines to produce robust and replicable results in migraine.
\end{abstract}

Keywords: Migraine, Voxel-based morphometry, Gray matter, Coordinate-based meta-analysis

\section{Background}

Migraine is a prevalent and disabling neurological disorder [1]. However, its pathophysiology remains poorly understood. Voxel-based morphometry (VBM) is a popular non-invasive magnetic resonance imaging (MRI) technique that enables us to investigate brain gray matter (GM) differences between groups [2]. In 2003,

\footnotetext{
* Correspondence: chyuanyc3y@163.com

${ }^{+}$Hai Cun Shi and Cong Hu Yuan contributed equally to this work. ${ }^{3}$ Department of Anesthesia and Pain Management, Affiliated Yancheng Hospital, School of Medicine, Southeast University, West Xindu Road 2\#, Yancheng, Jiangsu Province 224001, People's Republic of China Full list of author information is available at the end of the article
}

Matharu and colleagues performed the first whole-brain VBM study in migraine and found no significant GM alterations [3]. Since then, numerous VBM studies have been conducted. Recently, two VBM studies in migraine were published in The Journal of Headache and Pain $[4,5]$. One study by Liu and colleagues showed decreased GM volume in the right supramarginal gyrus and increased GM volume in the right cerebellar crus II in patients with highfrequency migraine relative to healthy controls [5]. The other study by Bonanno and colleagues reported different results and showed distinct patterns of GM abnormalities in migraine patients with and without aura relative to

(c) The Author(s). 2020 Open Access This article is licensed under a Creative Commons Attribution 4.0 International License, which permits use, sharing, adaptation, distribution and reproduction in any medium or format, as long as you give appropriate credit to the original author(s) and the source, provide a link to the Creative Commons licence, and indicate if changes were made. The images or other third party material in this article are included in the article's Creative Commons licence, unless indicated otherwise in a credit line to the material. If material is not included in the article's Creative Commons licence and your intended use is not permitted by statutory regulation or exceeds the permitted use, you will need to obtain permission directly from the copyright holder. To view a copy of this licence, visit http://creativecommons.org/licenses/by/4.0/ The Creative Commons Public Domain Dedication waiver (http://creativecommons.org/publicdomain/zero/1.0/) applies to the data made available in this article, unless otherwise stated in a credit line to the data. 
healthy controls [4]. Such results are noteworthy, however, reviewing the two and previous published studies, we found considerable variations of the VBM results. Spatially diverse brain regions with decreased and increased GM and null findings have been reported (Supplementary Table 1). The replicability and generalizability are increasingly concerned in neuroimaging research. It is interesting to know whether there is a reliable brain morphological signature for migraine, which may be of clinical and translational importance.

\section{Main text}

Coordinate-based meta-analysis (CBMA) is a powerful approach to quantitatively pool individual neuroimaging studies to identify consistent and reliable findings $[6,7]$. Several CBMA have been conducted to find consistent brain GM abnormalities in patients with migraine [8-11]. The first CBMA published online on March 15, 2015 using the activation likelihood estimation (ALE) approach included five VBM studies and found GM reductions in the middle and inferior frontal cortices in migraine [8]. Published on May 2nd, 2015, Dai et al. conducted the second CBMA using anisotropic effect size version of signed differential mapping (AES-SDM), which included nine VBM studies and showed consistent GM reductions in the posterior insular-opercular regions, prefrontal cortex, and anterior cingulate cortex [9]. Published on January 19, 2017, the third CBMA of eight VBM studies using ALE by Jia and Yu was performed, who found GM reductions in the bilateral inferior frontal gyri, right precentral gyrus, left middle frontal gyrus, and the left cingulate gyrus in migraine [10]. A most recent CBMA using ALE published as a medRxiv preprint on February 20, 2020 by Masson et al. included 27 VBM studies and detected no significant decrease of GM volume in migraine [11]. We noted that these CBMA studies also demonstrated divergent results. Several factors contribute to the divergences. First, the former three CBMA included relatively low numbers of VBM studies. It has been shown that at least 17 experiments in the ALE analysis would achieve reasonable power for statistical analysis [12]. Second, the algorithms for the CBMA have evolved. The old versions of ALE or use of uncorrected statistical threshold would yield spurious results [13]. The revised version of ALE applied in the recent CBMA used strict statistical threshold [11]. Third, differences of the algorithms applied in the AES-SDM and ALE would yield inconsistent results. ALE only included the studies with significant neuroimaging results. In contrast, AES-SDM quantitatively integrated both significant and null findings [14]. Fourth, variations in inclusion and exclusion criteria for the CBMA may also contribute to the inconsistences. According to the recent best-practice guidelines for CBMA, studies that applied region of interest (ROI)-based analysis or small-volume correction
(SVC) have to be excluded [6, 7]. Reviewing the studies included in the CBMA by Masson et al., several studies should not be included.

Recently, a new generation algorithms for CBMA, seed-based d mapping with permutation of subject images (SDM-PSI, https://www.sdmproject.com/), has been presented and recommended $[15,16]$. SDM-PSI makes significant improvements, such as use of standard voxelwise tests, standard permutation of subject images (PSI), unbiased estimation of effect sizes based on MetaNSUE algorithms, random-effects models, Freedman-Lanebased permutations, and threshold-free cluster enhancement (TFCE) statistics. These improvements avoid the drawbacks of the alternative procedures used in other current CBMA methods, thus making the results more accurate [15]. We therefore conducted an updated CBMA of available whole-brain VBM studies using SDM-PSI. After a comprehensive and careful literature search and screen, our CBMA finally included 32 wholebrain VBM studies (41 datasets) [3-5, 11, 17-44] involving a total of 1252 patients with migraine (988 females/ 264males, mean age 37.63 years) and 1025 healthy controls (794 females/231males, mean age 36.78 years). The present CBMA using SDM-PSI did not find evidence of consistent GM alterations in migraine (threshold-free cluster enhancement corrected, $p<0.05$ and cluster size $\geq 10$ voxels). Complementary analyses, such as sensitivity analysis, subgroup meta-analyses, and meta-regression analyses confirm the result robust. The details of the search strategy, study selection criteria, methods, the Tables regarding the demographic and clinical characteristics (Supplementary Table 1), imaging methodological information (Supplementary Table 2), and results of the SDM-PSI meta-analysis were provided in the Supplementary Materials.

The latest comprehensive CBMA with the largest number of whole-brain VBM studies reveals that there is no a brain morphological signature for migraine. Migraine is a heterogeneous disorder. VBM studies have shown that GM alterations are associated with attack frequency $[5,30,35,40]$, disease duration [21,35], disease severity [23], aura [4], migraine cycle (during or between attacks) [20], long-term outcomes [5], the number of tablets taken per month [19], and medication overuse [24]. In addition, age and female gender are potential confounding factors that may affect GM alterations in migraine $[9,45-47]$. Migraine is associated with higher rates of psychiatric disorders, such as major depression, bipolar disorder, and anxiety disorders [48]. However, these comorbidities have not been comprehensively assessed and controlled in previous VBM studies. In addition, sample sizes in most of the individual VBM datasets were under 50 participants per group. Underpowered studies with small sample sizes may produce 
unreliable results [49]. A software tool, called PowerMap has been developed to undertake statistical power calculation in neuroimaging studies [50]. Furthermore, it has been proposed that differences in MRI scanners, head coils, scanning parameters, preprocessing procedures, and statistical strategies may yield divergent results in individual VBM studies $[49,51]$. The inconsistences prevent us to obtain robust brain morphological features and limit the transdiagnostic effect in migraine. Lately, a more reliable technique called surface-based morphometry (SBM) was introduced to study groups' cortical thickness differences, which may provide more insights regarding brain morphology for migraine.

\section{Conclusions}

VBM investigations in migraine remain a heterogeneous field. The CMBA of available VBM studies found no evidence of consistent GM alterations in migraine. The quantitative evidence reveals that there is no brain morphological signature for migraine. The search for neuroimaging biomarkers for migraine is still on the way. Future VBM studies call for the control of potential confounding factors, such as the enrollment of well-characterized and homogeneous subtype samples with appropriate sample sizes, comprehensive assessment of comorbidities and medication status, and use of well-validated and standardized imaging protocols and processing and analysis pipelines (use of high field strength MRI with high spatial resolution, more recent software package, appropriate covariates in the statistical model, such as total intracranial volume or total GM volume, age, gender, and other comorbidity, and corrected thresholds for multiple comparisons) to produce robust and replicable results in migraine.

\section{Supplementary information}

Supplementary information accompanies this paper at https://doi.org/10. 1186/s10194-020-01158-7.

\section{Additional file 1}

\section{Abbreviations}

AES-SDM: Anisotropic effect size version of signed differential mapping; ALE: Activation likelihood estimation; CBMA: Coordinate-based meta-analysis; GM: Gray matter; MRI: Magnetic resonance imaging; ROI: Region of interest: SDM-PSI: Seed-based d mapping with permutation of subject images; SVC: Small-volume correction; VBM: Voxel-based morphometry

\section{Acknowledgements}

We thank all the authors of the included studies.

\section{Authors' contributions}

HCS and CHY designed the study. HZW, WHW and HCS performed the experiments. HZW and WHW analyzed the data. HZW, HCS, and CHY prepared the manuscript. All authors read and approved the final manuscript.

\section{Funding}

No funding supported this work.

\section{Availability of data and materials}

The datasets generated and/or analyzed during the current study are available from the corresponding author on reasonable request.

Ethics approval and consent to participate

Not applicable.

\section{Consent for publication}

Not applicable.

\section{Competing interests}

The authors declare that they have no competing interests.

\section{Author details}

${ }^{1}$ Department of Neurology, Kunshan Hospital, Affiliated to Jiangsu University, Kunshan, People's Republic of China. ${ }^{2}$ Department of Neurology, Affiliated Yancheng Hospital, School of Medicine, Southeast University, Yancheng, People's Republic of China. ${ }^{3}$ Department of Anesthesia and Pain Management, Affiliated Yancheng Hospital, School of Medicine, Southeast University, West Xindu Road 2\#, Yancheng, Jiangsu Province 224001, People's Republic of China.

Received: 1 May 2020 Accepted: 6 July 2020

Published online: 11 July 2020

\section{References}

1. Lerebours F, Boulanouar K, Barège M et al (2019) Functional connectivity of hypothalamus in chronic migraine with medication overuse. Cephalalgia. 39(7):892-899. https://doi.org/10.1177/0333102419833087

2. Ashburner J, Friston KJ (2000) Voxel-based morphometry--the methods. Neurolmage. 11(6 Pt 1):805-821. https://doi.org/10.1006/nimg.2000.0582

3. Matharu MS, Good CD, May A et al (2003) No change in the structure of the brain in migraine: a voxel-based morphometric study. Eur J Neurol 10(1):5357. https://doi.org/10.1046/j.1468-1331.2003.00510.x

4. Bonanno L, Lo Buono V, De Salvo S et al (2020) Brain morphologic abnormalities in migraine patients: an observational study. J Headache Pain 21(1):39. https://doi.org/10.1186/s10194-020-01109-2

5. Liu HY, Lee PL, Chou KH et al (2020) The cerebellum is associated with 2year prognosis in patients with high-frequency migraine. J Headache Pain 21(1):29. https://doi.org/10.1186/s10194-020-01096-4

6. Muller VI, Cieslik EC, Laird AR et al (2018) Ten simple rules for neuroimaging meta-analysis. Neurosci Biobehav Rev 84:151-161. https://doi.org/10.1016/j. neubiorev.2017.11.012

7. Tahmasian M, Sepehry AA, Samea F et al (2019) Practical recommendations to conduct a neuroimaging meta-analysis for neuropsychiatric disorders. Hum Brain Mapp 40(17):5142-5154. https://doi.org/10.1002/hbm.24746

8. Hu W, Guo J, Chen N et al (2015) A meta-analysis of voxel-based morphometric studies on migraine. Int J Clin Exp Med 8(3):4311-4319

9. Dai Z, Zhong J, Xiao P et al (2015) Gray matter correlates of migraine and gender effect: a meta-analysis of voxel-based morphometry studies. Neuroscience. 299:88-96. https://doi.org/10.1016/j.neuroscience.2015.04.066

10. Jia Z, Yu S (2017) Grey matter alterations in migraine: a systematic review and meta-analysis. Neuroimage Clin 14:130-140. https://doi.org/10.1016/j. nicl.2017.01.019

11. Masson R, Demarquay G, Meunier D et al (2020) Is migraine associated to brain anatomical alterations? New data and an ALE meta-analysis. medRxiv. https://doi.org/10.1101/2020.02.18.20024554

12. Eickhoff SB, Nichols TE, Laird AR et al (2016) Behavior, sensitivity, and power of activation likelihood estimation characterized by massive empirical simulation. Neuroimage. 137:70-85. https://doi.org/10.1016/j.neuroimage. 2016.04.072

13. Eickhoff SB, Laird AR, Fox PM et al (2017) Implementation errors in the GingerALE software: description and recommendations. Hum Brain Mapp 38(1):7-11. https://doi.org/10.1002/hbm.23342

14. Radua J, Rubia K, Canales-Rodriguez EJ et al (2014) Anisotropic kernels for coordinate-based meta-analyses of neuroimaging studies. Front Psychiatry 5:13. https://doi.org/10.3389/fpsyt.2014.00013

15. Albajes-Eizagirre A, Solanes A, Vieta E et al (2019) Voxel-based metaanalysis via permutation of subject images (PSI): theory and implementation for SDM. Neuroimage. 186:174-184. https://doi.org/10. 1016/j.neuroimage.2018.10.077 
16. Albajes-Eizagirre A, Solanes A, Fullana MA et al (2019) Meta-analysis of voxel-based neuroimaging studies using seed-based d mapping with permutation of subject images (SDM-PSI). J Vis Exp. (153)e59841:1-7. https:// doi.org/10.3791/59841

17. Celle S, Creac'h C, Boutet C et al (2018) Elderly patients with ongoing migraine show reduced gray matter volume in second somatosensory cortex. J Oral Facial Pain Headache 32(1):67-74. https://doi.org/10.1 1607/ofph.1866

18. Chanraud S, Di Scala G, Dilharreguy B et al (2014) Brain functional connectivity and morphology changes in medication-overuse headache: clue for dependence-related processes? Cephalalgia. 34(8):605-615. https:// doi.org/10.1177/0333102413519514

19. Chen WT, Chou KH, Lee PL et al (2018) Comparison of gray matter volume between migraine and "strict-criteria" tension-type headache. J Headache Pain 19(1):4. https://doi.org/10.1186/s10194-018-0834-6

20. Coppola G, Di Renzo A, Tinelli E et al (2015) Evidence for brain morphometric changes during the migraine cycle: a magnetic resonancebased morphometry study. Cephalalgia. 35(9):783-791. https://doi.org/10. 1177/0333102414559732

21. Coppola G, Petolicchio B, Di Renzo A et al (2017) Cerebral gray matter volume in patients with chronic migraine: correlations with clinical features. J Headache Pain 18(1):115. https://doi.org/10.1186/s10194-017-0825-z

22. Hougaard A, Amin FM, Arngrim N et al (2016) Sensory migraine aura is not associated with structural grey matter abnormalities. Neuroimage Clin 11: 322-327. https://doi.org/10.1016/j.nicl.2016.02.007

23. Hubbard CS, Khan SA, Keaser ML et al (2014) Altered brain structure and function correlate with disease severity and pain catastrophizing in migraine patients. eNeuro 1(1):e20.14. https://doi.org/10.1523/ENEURO.0006-14.2014

24. Lai TH, Chou KH, Fuh JL et al (2016) Gray matter changes related to medication overuse in patients with chronic migraine. Cephalalgia. 36(14): 1324-1333. https://doi.org/10.1177/0333102416630593

25. Linnman C, Catana C, Petkov MP et al (2018) Molecular and functional PETfMRI measures of placebo analgesia in episodic migraine: preliminary findings. Neuroimage Clin 17:680-690. https://doi.org/10.1016/j.nicl.2017.11.011

26. Lisicki M, D'Ostilio K, Coppola G et al (2018) Brain correlates of single trial visual evoked potentials in migraine: more than meets the eye. Front Neurol 9:393. https://doi.org/10.3389/fneur.2018.00393

27. Liu J, Lan L, Mu J et al (2015) Genetic contribution of catechol-Omethyltransferase in hippocampal structural and functional changes of female migraine sufferers. Hum Brain Mapp 36(5):1782-1795. https://doi.org/ 10.1002/hbm.22737

28. Mehnert J, May A (2019) Functional and structural alterations in the migraine cerebellum. J Cereb Blood Flow Metab 39(4):730-739. https://doi. org/10.1177/0271678X17722109

29. Messina R, Rocca MA, Colombo B et al (2018) Gray matter volume modifications in migraine: a cross-sectional and longitudinal study. Neurology. 91(3):e280-ee92. https://doi.org/10.1212/WNL. 0000000000005819

30. Neeb L, Bastian K, Villringer K et al (2017) Structural gray matter alterations in chronic migraine: implications for a progressive disease? Headache. 57(3): 400-416. https://doi.org/10.1111/head.13012

31. Niddam DM, Lai KL, Fuh JL et al (2016) Reduced functional connectivity between salience and visual networks in migraine with aura. Cephalalgia. 36(1):53-66. https://doi.org/10.1177/0333102415583144

32. Palm-Meinders $\mathbb{H}$, Arkink EB, Koppen $\mathrm{H}$ et al (2017) Volumetric brain changes in migraineurs from the general population. Neurology. 89(20): 2066-2074. https://doi.org/10.1212/WNL.0000000000004640

33. Rocca MA, Ceccarelli A, Falini A et al (2006) Brain gray matter changes in migraine patients with T2-visible lesions: a 3-T MRI study. Stroke. 37(7):17651770. https://doi.org/10.1161/01.STR.0000226589.00599.4d

34. Schmidt-Wilcke T, Ganssbauer S, Neuner T et al (2008) Subtle grey matter changes between migraine patients and healthy controls. Cephalalgia. 28(1): 1-4. https://doi.org/10.1111/j.1468-2982.2007.01428.x

35. Schmitz N, Admiraal-Behloul F, Arkink EB et al (2008) Attack frequency and disease duration as indicators for brain damage in migraine. Headache. 48(7):1044-1055. https://doi.org/10.1111/j.1526-4610.2008.01133.x

36. Tedeschi G, Russo A, Conte F et al (2016) Increased interictal visual network connectivity in patients with migraine with aura. Cephalalgia. 36(2):139-147. https://doi.org/10.1177/0333102415584360

37. Wang S, Wang H, Zhao D et al (2019) Grey matter changes in patients with vestibular migraine. Clin Radiol 74(11):898 e1-898 e5. https://doi.org/10. 1016/j.crad.2019.07.015
38. Wei HL, Zhou X, Chen YC et al (2019) Impaired intrinsic functional connectivity between the thalamus and visual cortex in migraine without aura. J Headache Pain 20(1):116. https://doi.org/10.1186/s10194-019-1065-1

39. Yang FC, Chou KH, Lee PL et al (2019) Patterns of gray matter alterations in migraine and restless legs syndrome. Ann Clin Transl Neurol 6(1):57-67. https://doi.org/10.1002/acn3.680

40. Yu Y, Zhao H, Dai L et al (2020) Headache frequency associates with brain microstructure changes in patients with migraine without aura. Brain Imaging Behav. https://doi.org/10.1007/s11682-019-00232-2

41. Zhang J, Wu YL, Su J et al (2017) Assessment of gray and white matter structural alterations in migraineurs without aura. J Headache Pain 18(1):74. https://doi.org/10.1186/s10194-017-0783-5

42. Lai KL, Niddam DM, Fuh JL et al (2020) Cortical morphological changes in chronic migraine in a Taiwanese cohort: surface- and voxel-based analyses. Cephalalgia. https://doi.org/10.1177/0333102420920005

43. Arkink EB, Schmitz N, Schoonman GG et al (2017) The anterior hypothalamus in cluster headache. Cephalalgia. 37(11):1039-1050. https:// doi.org/10.1177/0333102416660550

44. Zhe X, Gao J, Chen L et al (2020) Altered structure of the vestibular cortex in patients with vestibular migraine. Brain Behav 10(4):e01572. https://doi. org/10.1002/brb3.1572

45. Chong CD, Dodick DW, Schlaggar BL et al (2014) Atypical age-related cortical thinning in episodic migraine. Cephalalgia. 34(14):1115-1124. https://doi.org/10.1177/0333102414531157

46. Woldeamanuel YW, DeSouza DD, Sanjanwala BM et al (2019) Clinical features contributing to cortical thickness changes in chronic migraine - a pilot study. Headache. 59(2):180-191. https://doi.org/10.1111/head.13452

47. Maleki N, Linnman C, Brawn J et al (2012) Her versus his migraine: multiple sex differences in brain function and structure. Brain. 135(Pt 8):2546-2559. https://doi.org/10.1093/brain/aws175

48. Dresler T, Caratozzolo S, Guldolf K et al (2019) Understanding the nature of psychiatric comorbidity in migraine: a systematic review focused on interactions and treatment implications. J Headache Pain 20(1):51. https:// doi.org/10.1186/s10194-019-0988-x

49. Scott-Wittenborn N, Karadaghy OA, Piccirillo JF et al (2017) A methodological assessment of studies that use voxel-based morphometry to study neural changes in tinnitus patients. Hear Res 355:23-32. https://doi org/10.1016/j.heares.2017.09.002

50. Joyce KE, Hayasaka S (2012) Development of PowerMap: a software package for statistical power calculation in neuroimaging studies. Neuroinformatics. 10(4):351-365. https://doi.org/10.1007/s12021-012-9152-3

51. Celle S, Delon-Martin C, Roche F et al (2016) Desperately seeking grey matter volume changes in sleep apnea: a methodological review of magnetic resonance brain voxel-based morphometry studies. Sleep Med Rev 25:112-120. https://doi.org/10.1016/j.smrv.2015.03.001

\section{Publisher's Note}

Springer Nature remains neutral with regard to jurisdictional claims in published maps and institutional affiliations.
Ready to submit your research? Choose BMC and benefit from:

- fast, convenient online submission

- thorough peer review by experienced researchers in your field

- rapid publication on acceptance

- support for research data, including large and complex data types

- gold Open Access which fosters wider collaboration and increased citations

- maximum visibility for your research: over $100 \mathrm{M}$ website views per year

At $\mathrm{BMC}$, research is always in progress.

Learn more biomedcentral.com/submissions 